\title{
Self-Management Plans in Patients with Hereditary Angioedema: Strategies, Outcomes and Integration into Clinical Care
}

This article was published in the following Dove Press journal:

Journal of Asthma and Allergy

\section{Constance H Katelaris (1D \\ Immunology \& Allergy Unit, Department of Medicine, Campbelltown Hospital, Western Sydney University, Sydney, Campbelltown NSW 2560, Australia}

\begin{abstract}
Chronic conditions, whether genetic or acquired, impose a significant burden on health care systems with high utilisation of hospital and emergency department resources. Selfmanagement is increasingly recognised as one of the pillars in models of care for those with longterm medical conditions. Hereditary angioedema (HAE) is a rare genetic disorder inherited in an autosomal dominant fashion. It is characterised by the occurrence of unpredictable attacks of swelling (angioedema) affecting many body parts including subcutaneous tissues, the gut mucosa and the upper airway. For those affected, it is associated with a high burden of illness and poor quality of life as a result of its unpredictability and the threat of asphyxiation from upper airway oedema or severe pain from abdominal involvement. Prompt recognition and appropriate treatment are necessary to avoid the pain and suffering associated with attacks and to manage life-threatening laryngeal swellings that around $50 \%$ of HAE patients will experience in their lifetime. Since the early 2000 s, a number of very effective, albeit expensive, treatment options have become available, at least in some countries. Utilisation of these options within a written patient self-management plan provides the most satisfactory treatment outcomes and improves patient quality of life. Successful self-management depends on a productive partnership between patient and health care professional, with patient education the cornerstone of a successful outcome. This is a dynamic process, particularly in a condition such as HAE where frequency and severity of attacks may vary given different life circumstances.
\end{abstract}

Keywords: hereditary angioedema (HAE), treatment plan, self-management

\section{Introduction}

Self-management is increasingly recognised as a pillar in the models of care for chronic or long term conditions. ${ }^{1}$

Chronic conditions, whether genetic or acquired, impose a significant burden on health care systems with high utilisation of hospital and emergency department resources. Co-ordinating optimal health care by incorporating self-management plans is important to minimise the impact of these conditions at individual, community and health system levels. ${ }^{2}$

This paper will address the application of self-management principles and the use of a self-management treatment plan in Hereditary angioedema (HAE), a rare genetic disorder.

\section{HAE Background}

HAE is characterised by the occurrence of unpredictable attacks of swelling affecting many body parts. For those affected, it is associated with a high burden 
of illness and poor quality of life as a result of its unpredictability and the threat of asphyxiation from upper airway oedema or severe pain from abdominal involvement. ${ }^{3}$

HAE is inherited as an autosomal dominant disorder characterised by low or dysfunctional levels of C $1 \mathrm{INH}$. The abnormality is in the SERPING1 gene mapped to chromosome 11q12-q13.1. Over 700 mutations have been described. ${ }^{4}$ While the majority of those affected inherit the disorder, about $25 \%$ have a spontaneous mutation so they lack the characteristic family history. ${ }^{5}$

There are three types described: HAE type 1 (HAE-1) is the most common and results from abnormalities in one of the gene alleles producing low levels of $\mathrm{C} 1 \mathrm{INH}$; HAE type 2 (HAE-2) may have normal or elevated $\mathrm{C} 1 \mathrm{INH}$ levels but the protein is dysfunctional. A third type has been described that has normal levels of functional $\mathrm{C} 1$ INH (HAE- nC1INH). ${ }^{6}$ In some with this variant, other genetic mutations have been described, including mutations of factor XII, plasminogen, angiopoietin 1 and kininogen 1 genes. $^{7-10}$

$\mathrm{C} 1 \mathrm{INH}$ is a key regulatory molecule in the complement, contact and coagulation pathways. Low or defective levels of $\mathrm{C} 1 \mathrm{INH}$ result in unchecked complement activation as well as abnormalities in other pathways. Among its actions, C1 INH inhibits kallikrein. Bradykinin, a key mediator in the kallikrein system is responsible for capillary leakage causing the swelling experienced by those with HAE. Increased blood bradykinin levels have been associated with clinical flares. ${ }^{11,12}$

The diagnosis of the classical HAE types depends on the appropriate clinical history and the finding of low $\mathrm{C} 4$ levels, low or functionally abnormal C 1 INH levels and normal C1q. ${ }^{13}$ Genotyping may be required, particularly for accurate diagnosis of HAE-nC1INH patients. ${ }^{14}$

HAE has been described in all racial groups occurring in $1: 50,000-1: 150,000$ of the population. ${ }^{15}$ Males and females are equally affected but the condition tends to be more severe in females. While the defect is present at birth, neonatal swelling is rare. About $50 \%$ of cases present under 10 years with the majority of affected individuals experiencing swellings by late childhood and early teen years. ${ }^{16}$ Episodes of abdominal pain in children may be easily overlooked or blamed on other more common conditions. Swellings may occur throughout life but tend to lessen in later life. ${ }^{16}$

Clinically, individuals with HAE experience angioedema attacks (non-pruritic, non-pitting swelling) involving subcutaneous tissues, intestinal wall and the upper airway. About $50 \%$ of HAE patients experience at least one laryngeal attack in their lives and before effective therapy, about $30 \%$ of HAE patients died from asphyxiation. ${ }^{17}$

A number of triggers for attacks are identified by individuals, the most common being trauma and stress, but the majority of attacks are truly random and unpredictable. ${ }^{11}$ Some individuals experience a transient, non- pruritic lacy rash called erythema marginatum as a prelude to a swelling although others may develop this appearance without subsequent swelling. ${ }^{18,19}$

The true burden of having HAE comes from several aspects. Laryngeal attacks are potentially life-threatening and must be treated early and effectively, however if definitive treatment is not available, tracheotomy may be required as a life-saving procedure. Abdominal attacks cause much misery as they are painful and may resemble a bowel obstruction. The abdominal pain is caused by oedema of the mucosa of any part of the gastrointestinal tract. Many patients have undergone unnecessary laparotomies in the past when the diagnosis has been missed. Cutaneous swellings, particularly if they involve the hands, feet, face and genitals, can interfere substantially with daily life and may also be painful.

Frequency of attacks varies greatly between individuals and within an individual's lifetime. There may be periods of very frequent attacks, particularly at times of psychological and physical stress; at other times, the swellings may occur uncommonly. Angioedema due to HAE differs from allergic angioedema in that it usually has a slow onset and offset and may last several days. It does not respond to usual treatments such as antihistamines, oral corticosteroids or adrenaline used to manage angioedema caused by an allergy. ${ }^{20}$

The unpredictable nature of attacks impacts individuals' quality of life substantially as it is difficult to plan ahead or make commitments for fear of an attack. Many of those with HAE relate significant interruption to schooling and career advancement because of their condition. ${ }^{21}$

\section{HAE Management Strategies}

A cornerstone of management in HAE is understanding the need for early treatment of acute attacks with readily available specific therapies. Early therapy results in shorter time to resolution of symptoms and shorter total attack duration regardless of attack severity ${ }^{22}$. Replacing $\mathrm{C} 1 \mathrm{INH}$ or inhibiting activated plasma kallikrein or bradykinin have become the mainstay of acute therapy for HAE since the early 2000s, at least in countries where these options are available. The desirability for prompt recognition and treatment of an acute 
attack are major reasons why self-management has been promoted for HAE patients.

When attack rates are frequent enough to impinge on daily life and impair quality of life, consideration should be given to long- term treatment, or prophylaxis (LTP). ${ }^{20}$ A third treatment strategy is the use of short- term prophylaxis (STP), where prophylactic medication is given in the short- term to protect the individual from an increased risk of an attack when undergoing procedures known to precipitate attacks. These include invasive dental work and intubation for surgery when there is a substantially increased risk of inducing a life -threatening laryngeal attack. ${ }^{20}$

Until the early 2000's few treatment options were available to manage this condition. For acute attacks replacement treatment with a blood product, $\mathrm{C} 1$ INH concentrate, has been available in some countries since the 1980's although in most its use was very restricted to severe attacks only. It is administered intravenously so patients presented to hospital emergency departments for infusions. This usually entailed a number of delays and patients often encountered barriers to obtaining this specific therapy. Otherwise, only supportive therapy was available with analgesia, fluid replacement and anti -emetics.

Acute attack management was dramatically changed with the advent of the specific bradykinin receptor 2 antagonist, icatibant. This is administered by subcutaneous injection and was shown to be highly efficacious in three pivotal clinical trials. $^{23}$

Its introduction into clinical practice resulted in major positive impacts on quality of life of HAE patients as selfadministration is easily taught, the treatment is portable and very effective, and its availability placed management decisions firmly into the hands of patients no longer having to tolerate delays and lack of understanding in emergency departments. $^{24-26}$

Ecallantide is a small, 60-amino acid, recombinant protein inhibitor of plasma kallikrein, which was first approved by the FDA in December 2009 for treatment of acute attacks of HAE in patients and is now approved for patients 12 years and over as a subcutaneous injection. It inhibits kallikrein by blocking the plasma-binding site, thereby stopping production of bradykinin and, consequently, its effects. ${ }^{27}$ While it is available in the USA use in other parts of the world is limited. There have been cases of anaphylaxis reported with its use.

Craig et $\mathrm{al}^{28}$ conducted a study that specifically examined anaphylaxis associated with ecallantide. The study included 230 individuals treated with ecallantide and eight $(3.5 \%)$ had anaphylactic reactions within an hour of receiving ecallantide, none with the first dose.

For those with frequent attacks, two drugs, tranexamic acid and danazol, have been available for many years in most countries for LTP. Neither are completely effective and danazol in particular, causes significant side effects in many patients, particularly after long-term use. ${ }^{29,30}$

Since 2008, treatment options have expanded and improved. C1 INH concentrate is available as plasmaderived products (Berinert (CSL Behring) and Cinryze (Shire,Takeda)) and as a recombinant $\mathrm{C} 1 \mathrm{INH}-$ Ruconest (Pharming Group/Salix Pharmaceuticals).This is a human recombinant $\mathrm{C} 1$ esterase inhibitor purified from the milk of transgenic (genetically modified) rabbits. The drug is intended to restore the level of functional $\mathrm{C} 1$ esterase inhibitor in the plasma, thereby treating an acute attack of swelling. ${ }^{31}$ These products, administered intravenously, are used for both acute treatment and for those requiring LTP when $\mathrm{C} 1 \mathrm{INH}$ concentrate is administered at frequent regular intervals in an attempt to prevent swellings. Many patients have been taught self-cannulation so these treatments can be on hand in the home for prompt administration in the event of an acute attack or for convenient prophylactic therapy.

More recently a subcutaneously administered form of Berinert has become available for LTP. Clinical studies suggest that this product has greater efficacy and it is certainly more convenient for patients to administer. ${ }^{32}$

The most recent addition to HAE therapeutic options is lanadelumab, a monoclonal antibody directed against kallikrein. It is administered subcutaneously once a fortnight making it the most convenient option for LTP to date. In clinical trials it has been highly efficacious and has a good side effect profile. It is now registered in several countries and is in clinical use in the USA and in some European countries. $^{33}$

All the currently approved therapies are delivered by injection or infusion. An orally delivered, small molecule inhibitor of plasma kallikrein is in development and will offer the opportunity to improve treatment for the disease by making it more convenient for patients to take therapies. ${ }^{34}$

\section{HAE Self-Management}

Many features of HAE make it a condition very suitable for patient self-management. Being a rare condition, most medical practitioners will not have seen a patient with the condition and many know very little about it. In this situation, the patient becomes the expert and is able to 
guide medical staff regarding appropriate treatment for symptoms. A knowledgeable patient is advantageous for managing all chronic medical conditions but this is particularly pertinent for a rare disease when medical professionals are unlikely to have encountered the condition in their experience.

As a strategy for managing chronic conditions, selfmanagement moves beyond education to teaching individuals to be actively involved in treatment decisions, problemsolving and appropriate medication administration. ${ }^{35}$ Most importantly, self-management plans allow patients to maintain independence and to have a degree of control over a chronic condition, thus assisting in improving quality of life. The selfmanagement process involves problem-solving, decision making, utilisation of medication and other treatments, partnering with health care professionals and undertaking regular reviews and knowledge enhancement. ${ }^{36}$

There are various types of self-management plans and these may be led by a medical person or the patient. Whichever way they are devised, the goal is to empower the individual with knowledge and skills to utilise medications or strategies in the most advantageous manner to minimise impacts of the condition or symptoms on lifestyle. Self-management plans can be devised for different settings to be used by various individuals involved in care delivery. Instructions may be for self-management or for a family member or care- giver to administer treatment, or for use by emergency department staff when attending a patient with a rare or life-threatening emergency. Depending on the particular medical condition, content and design of a plan will differ.

Several steps are necessary in devising a selfmanagement plan independent of the chronic condition experienced by the patient. Foremost is the individual's own values and preferences. Detailed discussions about the particular medical condition, the various treatment options available and their effects and side effects need to be discussed so informed decisions can be made. The medical practitioner needs to assess the knowledge, skills and attitudes of the patient to determine the best way forward. Careful discussion of how the plan is to be used with checks on patient understanding performed. Follow up visits for monitoring successful implementation, identification of any barriers or problems and continuing education, are necessary. Appropriate notification of the care plan in the medical file is the final step in the process. ${ }^{2}$

Self-management plans have been part of routine practice for patients with chronic diseases such as asthma and diabetes for many years. More recently there have been very effective management plans devised for those at risk of anaphylaxis so these individuals are coached in early recognition of potentially life-threatening signs and symptoms and early institution of emergency treatment with appropriate drugs. ${ }^{37}$ In many disease states, self-management programs are proven models for successful chronic disease management leading to fewer hospital presentations and improved quality of life and are now adopted as standard practice in many countries including the USA, Australia, United Kingdom, Canada and New Zealand. ${ }^{38}$

The Cochrane Collaboration has conducted evidencebased reviews of a number of self-management programs such as those for asthma with positive findings supporting their role in clinical practice. ${ }^{39}$

As outlined above, treatment options for those with HAE have increased considerably over the last decade, at least in some countries. There are various treatment options available for acute management and LTP so patients do have some choice in how to manage their condition. Knowing how to manage HAE when there is a need for invasive dental work or surgery is also very important. The goals of devising individual management plans is to decrease morbidity, prevent mortality and improve quality of life by giving a degree of control over an unpredictable condition. There are various steps in the process of constructing such a plan, beginning with patient education consisting of a careful discussion about available treatment options, their effects and side effects. Ideally, one or more family members or a carer should be involved in this process as well. Writing the action plan, discussing its implementation and checking that the patient understands it is vital. Monitoring its use, gauging the appropriateness of actions taken, and adjusting the plan according to the patient's attack rate are the final steps for ensuring maximal benefit. Self-management plan construction and implementation should be seen as a dynamic process that adapts to individual needs at various times.

\section{Specific Components of HAE Management Plans}

The first step in the HAE self-management process is to provide education and written information that can be presented to emergency department staff if a patient needs to present to hospital. This information may be in the form of a letter,emergency card, website contact or documents on a device. This is a rare condition and the 
vast majority of emergency department staff will not have had the opportunity to treat an HAE attack.

Secondly, the plan must incorporate details about treating an acute attack. International guidelines state that all patients should have at least two doses of acute treatment on hand at all times. ${ }^{20}$ Other information to record includes when to treat (early in the attack; ideally within the first few hours); when to repeat treatment; what to do when on demand treatment is not available and how to handle the medication including appropriate storage instructions. Specific instructions of what is needed for travel, school or the workplace is also necessary.

Some but not all HAE patients may require LTP. How this need is determined varies and depends on many factors including local regulations. In most countries, danazol is available but discussions concerning effects and side effects need to be undertaken before a patient can make an informed choice. In many countries C 1 INH concentrate for IV administration is the other option, and likewise, careful discussion concerning its administration, storage and other logistics need to be had with the patient. Very recently two more convenient and highly efficacious forms of LTP have become available at least in some countries. Both Berinert SC (Haegarda) and lanadelumab (Takzhyro) are administered subcutaneously making them far more attractive options. In ideal circumstances, factors other than numerical attack rates must be considered in gauging the need for LTP, including impact on a particular patient's life, distance from emergency medical treatment centres, and presence of particular stressors. Patient preferences, success with on demand treatments and ease of venous access are also considerations. ${ }^{40}$

Adaptation of HAE management plans will be necessary for special situations such as pregnancy and lactation, and for paediatric patients.

\section{Conclusions}

Chronic diseases including genetic disorders impose significant burden on both patients and the health care systems. Engaging such patients in self-management to optimally utilise therapies is an important step in cost containment and improved health care outcomes. HAE, a rare genetic disorder for which several new, very expensive, treatment modalities are now available, provides a good example of a life-long, chronic condition where an educated patient, trained in appropriate self-management, can experience better outcomes and quality of life. Patients with rare conditions become the experts in management of their condition and with the appropriate tools can optimise their own management while providing useful information for non-expert health professionals.

The basic principles of HAE self-management are patient and carer education tailored to individual knowledge, skills and attitudes, written treatment plans and regular review and revision depending on changing circumstances. Future needs in HAE management include patient-centred research to assess effectiveness and adherence to written treatment plans and to further refine design to accommodate evolving treatment options and patient needs.

\section{Disclosure}

Prof Katelaris has conducted clinical trials for CSL Behring, Shire/Takeda and Biocryst for which her institution has received funds.

She has received honoraria for presentations on HAE and for advisory board participation. She has participated in the writing of International Guidelines for HAE management and is a Board member of HAE Australasia. The author reports no other conflicts of interest in this work.

\section{References}

1. Wagner EH, Austin BT, Davis C, et al. Improving chronic illness care: translating evidence into action. Health Aff (Millwood). 2001;20 (6):64-78. doi:10.1377/hlthaff.20.6.64

2. National Institute for Health and Care Excellence. Medicines optimisation.The Safe and Effective Use of Medicines to Enable the Best Possible Outcomes. March 2015. NICE Guideline 5.

3. Cicardi M, Aberer W, Banerji A, et al. Classification, diagnosis, and approach to treatment for angioedema: consensus report from the hereditary angioedema international working group. Allergy. 2014;69(5):602-616. doi:10.1111/all.12380

4. Ponard D, Gaboriaud C, Charignon C, et al. SERPING1 mutation update: mutation spectrum and $\mathrm{C} 1$ inhibitor phenotypes. Hum Mutat. 2020;41(1):38-57. doi:10.1002/humu.23917

5. Zanichelli A, Longhurst H, Maurer M, et al. Misdiagnosis trends in patients with hereditary angioedema from the real-world clinical setting. Ann Allergy Asthma Immunol. 2016;117(4):394398. doi:10.1016/j.anai.2016.08.014

6. Bruce L. Hereditary angioedema with normal C1 inhibitor: four types and counting. $J$ Allergy Clin Immun. 2018;141(3):884-885. doi:10.1016/j.jaci.2018.01.015

7. Bork K, Wulff K, Hardt J, et al. Hereditary angioedema caused by missense mutations in the factor XII gene: clinical features, trigger factors, and therapy. J Allergy Clin Immunol. 2009;124(1):129-134. doi:10.1016/j.jaci.2009.03.038

8. Bork K, Wulff K, Steinmeuller-Magin L, et al. Hereditary angioedema with a mutation in the plasminogen gene. Allergy. 2018;73 (2):442-450. doi:10.1111/all.13270

9. Germenis A, Loules G, Zamanakou M, et al. On the pathogenicity of the plasminogen K330E mutation for hereditary angioedema. Allergy. 2018;73(8):1751-1753. doi:10.1111/all.13324

10. Bork K, Wulff K, Rossmann H, et al. Hereditary angioedema cosegregating with a novel kininogen 1 gene mutation changing the N-terminal cleavage site of bradykinin. Allergy. 2019;74(12):2479. doi:10.1111/all.13869 
11. Zuraw BL. Clinical practice. Hereditary angioedema. $N$ Engl J Med. 2008;359(10):1027-1036. doi:10.1056/NEJMcp0803977

12. Davis AE. Mechanism of angioedema in first complement component inhibitor deficiency. Immunol Allergy Clin North Am. 2006;26 (4):633-651. doi:10.1016/j.iac.2006.08.003

13. Craig T, Aygören-Pürsün E, Bork K. WAO guidelines for the management of hereditary angioedema. WAO J. 2012;5(12):182-199. doi:10.1097/WOX.0b013e318279affa

14. Germenis AE, Margaglione M, Pesquero JB, et al. International consensus on the use of genetics in the management of hereditary angioedema. $J$ Allergy Clin Immunol Pract. 2019;8:901-911. doi:10.1016/j.jaip.2019.10.004

15. Bygum A. Hereditary angio-oedema in Denmark: a nationwide survey. Brit J Dermatol. 2009;161(5):1153-1158. doi:10.1111/ j.1365-2133.2009.09366.x

16. Bork K, Meng G, Staubach P, Hardt J. Hereditary angioedema: new findings concerning symptoms, affected organs, and course. $\mathrm{Am}$ $J$ Med. 2006;119(3):267-274. doi:10.1016/j.amjmed.2005.09.064

17. Bork K, Siedlecki K, Bosch S, et al. Asphyxiation by laryngeal edema in patients with hereditary angioedema. Mayo Clin Proc. 2000;75(4):349-354. doi:10.4065/75.4.349

18. Starr JC, Brasher GW, Rao A, Posey D. Erythema marginatum and hereditary angioedema. South Med J. 2004;97(10):948-950. doi:10.1097/01.SMJ.0000140850.22535.FA

19. Farkas H, Harmat G, Fáy A, et al. Erythema marginatum preceding an acute oedematous attack of hereditary angioneurotic oedema. Acta Derm Venereol. 2001;81(5):376-377. doi:10.1080/000155501317140188

20. Maurer M, Magerl M, Ansotegui I, et al. The international WAO/ EAACI guideline for the management of hereditary angioedema - the 2017 revision and update. World Allergy Organ J. 2018;11(1):1. doi:10.1186/s40413-017-0180-1

21. Lumry WR, Castaldo AJ, Vernon MK, Blaustein MB, Wilson DA, Horn PT. The humanistic burden of hereditary angioedema: impact on health-related quality of life, productivity, and depression. Allergy Asthma Proc. 2010;31(5):407-414. doi:10.2500/aap.2010.31.3394

22. Craig TJ, Rojavin MA, Machnig T, Keinecke H-O, Bernstein JA. Effect of time to treatment on response to $\mathrm{C} 1$ esterase inhibitor concentrate for hereditary angioedema attacks. Ann Allergy Asthma Immunol. 2013;111(3):211-215. doi:10.1016/j.anai.2013.06.021

23. Cicardi M, Banerji $A$, Bracho $F$, et al. Icatibant, a new bradykinin-receptor antagonist, in hereditary angioedema. $N$ Engl J Med. 2010;363(6):532-541. doi:10.1056/NEJMoa0906393

24. Banerji A. The burden of illness in patients with hereditary angioedema. Ann Allergy Asthma Immunol. 2013;111(5):329-336. doi:10.1016/j.anai.2013.08.019

25. Hernandez Fernandez de Rojas D, Ibanez E, Longhurst $\mathrm{H}$, et al. Treatment of HAE attacks in the icatibant outcome survey: an analysis of icatibant self-administration versus administration by health care professionals. Int Arch Allergy Immunol. 2015;167(1):2128. doi: $10.1159 / 000430864$
26. Bygum A, Andersen KE, Mikkelsen CS. Self-administration of intravenous $\mathrm{C} 1$-inhibitor therapy for hereditary angioedema and associated quality of life benefits. Eur J Dermatol. 2009;19(2):147-151. doi: $10.1684 /$ ejd.2008.0603

27. Firszt R, Duffey H. Management of acute attacks of hereditary angioedema: role of ecallantide. J Blood Med. 2015;6:115-119. doi:10.2147/JBM.S66825

28. Craig TJ, Li HH, Riedl M, et al. Characterization of anaphylaxis after ecallantide treatment of hereditary angioedema attacks. J Allergy Clin Immunol Pract. 2015;3(2):206-212. doi:10.1016/j.jaip.2014.09.001

29. Zuraw BL, Davis DK, Castaldo AJ, Christiansen SC. Tolerability and effectiveness of 17- $\alpha$-alkylated androgen therapy for hereditary angioedema: a re-examination. J Allergy Clin Immunol Pract. 2016;4(5):948-955. doi:10.1016/j.jaip.2016.03.024

30. Bork K, Bygum A, Hardt J. Benefits and risks of danazol in hereditary angioedema: a long-term survey of 118 patients. Annals of Allergy, Asthma \& Immunology. 2008;100(2):153-161. doi:10.1016/ S1081-1206(10)60424-3

31. Paspe Cruz M. Conestat alfa (ruconest). first recombinant $\mathrm{C} 1$ esterase inhibitor for the treatment of acute attacks in patients with hereditary angioedema. Pharm Ther. 2015;40(2):109-111.

32. Craig T, Longhurst H, Cicardi M, Zuraw B. Safety and efficacy of long-term subcutaneous $\mathrm{C} 1$-inhibitor replacement therapy for prevention of hereditary angioedema attacks. Ann Allergy Asthma Immunol. 2018;121(5):S34. doi:10.1016/j.anai.2018.09.107

33. Banerji A, Riedl MA, Bernstein JA, et al. Effect of lanadelumab compared with placebo on prevention of hereditary angioedema attacks. JAMA. 2018;320(20):2108. doi:10.1001/jama.2018.16773

34. Aygören-Pürsün E, Bygum A, Grivcheva-Panovska V, et al. Oral plasma kallikrein inhibitor for prophylaxis in hereditary angioedema. $N$ Engl J Med. 2018;379(4):352-362. doi:10.1056/ NEJMoa1716995

35. Grady PA, Gough L. Self-management: a comprehensive approach to management of chronic conditions. Am J Public Health. 2014;104(8): e25-e31. doi:10.2105/AJPH.2014.302041

36. Lorig KR, Mazonson PD, Holman HR. Evidence suggesting that health education for self-management in patients with chronic arthritis has sustained health benefits while reducing health care costs. Arthritis Rheum. 1993;36(4):439-446. doi:10.1002/art.1780360403

37. www.allergy.org.au > anaphylaxis > ascia-action-plan-for-anaphylaxis

38. Dowrick C, Dixon-Woods M, Holman H, Weinman J. What is chronic illness? Chronic Illn. 2005;1(1):1-6. doi:10.1177/ 17423953050010010901

39. Gibson PG, Powell H, Coughlan J, et al. Self-management education and regular practitioner review for adults with asthma. Cochrane Database Syst Rev. 2003;1:CD001117.

40. Betschel S, Badiou J, Binkley K, et al. The international/Canadian hereditary angioedema guideline. Allergy Asthma Clin Immunol. 2019;15(1):72. doi:10.1186/s13223-019-0376-8

\section{Publish your work in this journal}

The Journal of Asthma and Allergy is an international, peer-reviewed open-access journal publishing original research, reports, editorials and commentaries on the following topics: Asthma; Pulmonary physiology; Asthma related clinical health; Clinical immunology and the immunological basis of disease; Pharmacological interventions and new therapies. The manuscript management system is completely online and includes a very quick and fair peer-review system, which is all easy to use. Visit http://www.dovepress.com/testimonials.php to read real quotes from published authors. 